\title{
Mal-estar da adolescência: transição identitária e contextos de interação social entre cultura e educação
}

\begin{abstract}
Resumo: A investigação destina-se à compreensão dos vários significados sociais que se concretizam através da sistematização do conhecimento, dentro dos próprios lugares de produção, nas agências de socialização, em contextos reais de interação, nos enlaces entre fatores individuais e fatores normativos, entre representações e cultura (mediação simbólica), bem como à análise dos conteúdos descritivos da representação, da rede dos significados sociais ligados aos contextos interativos e situacionais de ativação e reprodução dos sistemas de crenças. O seu objetivo é chegar a conhecer a percepção que os adolescentes têm da sua própria condição nos seus âmbitos de vida, procurando entender os elementos que caracterizam o mal-estar inerente à adaptação à mudança, seja esta pessoal ou contextual. O autor tenta destacar as representações sociais espalhadas pelo imaginário coletivo dos jovens e que são influenciadas pelo ambiente cultural de referência, a fim de entender como este mundo é percebido, conhecido, compreendido, encarado e comunicado durante a fase de transição identitária da adolescência. O instrumento escolhido para esse fim foi um questionário semiestruturado, que foi submetida a uma amostra de 200 adolescentes, que indaga os vários âmbitos de vida e as representações, tendo em conta a valência sócio-relacional e emotivo-afetiva das variáveis contextuais que entram no jogo da transição identitária.
\end{abstract}

Palavras-chave: Adolescência. Mal-estar. Contextos Culturais. Educação.

\section{Introdução teórica}

Ao longo da análise do mal-estar psicossocial ligado à transição identitária do adolescente, numa perspectiva histórico-cultural (VYGOTSKIJ, 1962) e socioconstrutivista (BRUNER, 1986, 1997; COLE, 1996), a atenção focalizou-se inevitavelmente nos aspectos representativos, nos momentos históricos e nas características dos contextos culturais nos quais se enfrentam as tarefas de desenvolvimento (HAVIGHURST, 1952).

A relação entre construção da identidade e psicologia histórico-cultural pode ser entendida, na afirmação, de acordo com a qual "a natureza psicológica do ser humano representa o conjunto das relações sociais transportadas no interior e transformadas em funções da personalidade e formas da sua estrutura" (VYGOTSKIJ, 1981, p. 202). A construção da identidade inclui atravessar crises caracterizadas por conflitos (ERIKSON, 1968) e, na adolescência, tal construção explica-se concretamente pelas interações sociais, interiorizando as experiências interpessoais e ativando os níveis de
Oreste Fasano

Universidade de Salermo, Itália ofasano@unisa.it 
funcionamento mental na construção funcional da personalidade (VYGOTSKIJ, 1934). Na construção da identidade atribui-se valor ao papel das interações sociais (e da comunicação através da linguagem), pois as funções mentais superiores originam-se e tomam forma mesmo no interior das relações interpessoais.

A relação entre construção da identidade e psicologia cultural pode ser explicada em vários níveis. O primeiro nível leva em consideração as mudanças biológicas e de maturação que envolvem os adolescentes em relação aos vários contextos socioculturais de proveniência e às atribuições de significado socialmente partilhadas, pois os indivíduos interiorizam o sistema cultural no qual se encontram e a natureza do desenvolvimento humano é essencialmente social (BRUNER, 1990). Através do desenvolvimento psicossexual, o adolescente experimenta novas sensações e emoções enquanto vive experiências significantes e importantes para a construção da sua identidade: interpretadas e redefinidas em relação aos significados atribuídos e atribuíveis pelos contextos sociais aos quais pertencem (FASANO, 2007). Relativamente a este processo de interpretação da experiência individual e da realidade da vida, do Eu e dos outros, os indivíduos constroem o seu conhecimento do mundo e modificam, por meio de um constante feedback, as suas atitudes futuras. De acordo com Bruner (1983), que concebeu o desenvolvimento como uma progressiva melhoria das potencialidades da criança através do papel desempenhado pela experiência das relações interpessoais, tais sistemas culturais são formados por concepções, valores, crenças e símbolos, capazes de assumir significados condicionantes, enquanto são transmitidos de uma geração para outra através do instrumento da linguagem, que permite conhecer, interpretar e negociar os significados da realidade.

O segundo nível está ligado ao estudo da adolescência que não pode ser separado das mudanças dos hábitos de vida e da evolução tecnológica. Em nível social e cultural, não se pode deixar de ter em conta as conquistas tecnológicas e as mutações que têm a ver com os atuais hábitos de vida: influências macrossistêmicas que se refletem em todos os níveis contextuais do sistema de vida. Os instrumentos tecnológicos caraterizam cada vez mais os processos de aprendizagem também em contexto escolar, transformando as modalidades de apresentação e a transmissão do conhecimento e do saber. O próprio instrumento da narração está a ser subme- 
tido a uma metamorfose, quer em termos comunicativos, quer culturais (FASANO, 2009). O "Eu transacional", ao qual Bruner se refere, é construído por meio da troca comunicativa interpessoal, ou seja, nas interações significantes entre pessoas que comunicam em situações e contextos específicos. A narração autobiográfica permite reorganizar a história individual das pessoas, exprimindo ao mesmo tempo o componente emotivo e a cognitivo, a fim de atribuir um sentido à própria história de vida. Trata-se de uma das modalidades que os sujeitos numa condição de transição utilizam para conseguir atribuir significado às suas experiências de mudança (MCADAMS, 1996). De acordo com Bruner (1986), o pensamento narrativo, utilizado na reconstrução autobiográfica, permite ligar o cenário da ação ao cenário da consciência modelando, desta forma, a complexa natureza do processo de construção da identidade que "tem em conta todos os níveis de funcionamento mental” (ERIKSON, 1968). Neste sentido, torna-se difícil imaginar uma mente capaz de organizar uma narração sem referenciar, de alguma forma, uma memória cultural partilhada, coletiva, tal como evidenciado por uma vasta tradição de estudos (SKOWRONSKI; WALKER, 2004; VYGOTSKIJ, 1962) que nos leva a uma reflexão sobre a multiplicidade de variáveis que influenciam o desenvolvimento e a construção da identidade.

\section{Objetivos da pesquisa}

A pesquisa teve como objetivo investigar, através do preenchimento de um questionário, as representações sociais espalhadas pelo imaginário coletivo dos jovens e que são influenciadas pelo seu ambiente cultural de referência (BRUNER, 1990; MOSCOVICI, 1984; VYGOTSKIJ, 1981), a fim de se entender como este mundo é percebido, conhecido, compreendido, encarado e comunicado durante a fase de transição identitária da adolescência. ${ }^{1}$ O conhecimento, uma vez que é o fruto dos significados produzidos pela capacidade de partilhar crenças, valores, atividades e atitudes, pode considerar-se inextricavelmente ligado ao contexto. Nesse sentido, sob um ponto de vista estritamente metodológico, podemos evidenciar uma explícita referência à dimensão narrativa e culturalista dos processos de construção identitária (BRUNER, 1990, 2009; IANNACCONE; SMORTI, 2009; SMORTI, 1997) entendida como modalidade que os indivíduos privilegiam para atribuir sentido e
10 modelo teórico-empírico de pesquisa das representações sociais é dotado de um sistema multidimensional de perspectivas destinado ao entendimento de fatores e processos implicados na gênese do pensamento social, ou seja, daquelas estruturas e mentalidades, estereótipos, opiniões, crenças que participam na formação de métodos-conteúdos de pensamento socializados e partilhados (DE ROSA, 1995). 
(2) O perfil socioanagráfico revela que o nível de instrução dos pais é geralmente médio-baixo: estes encontram-se prevalentemente incluídos nas categorias "ensino básico" e "secundário", com uma pequena percentagem de "licenciados". Os entrevistados vivem em famílias caracterizadas por um rendimento pouco elevado e que na maioria dos casos mantêm o esquema tradicional: o pai trabalha enquanto a mãe se dedica ao cuidado da casa e da família

(3) Para aprofundar as características sociais do território e dos seus habitantes, consultem Monda P. e Buono T. (2011). significado, organizar o conhecimento e partilhar a sua experiência e interpretação da realidade.

\section{Participantes e metodologia}

A amostra da pesquisa foi composta por 200 estudantes, divididos em dois grupos, formados respectivamente por 100 rapazes e 100 meninas, entre os 16 e os 18 anos de idade, provenientes dos municípios do Âmbito territorial Salerno 1 (Plano de Zona S1-Vale do Sarno) que preencheram um questionário anônimo. ${ }^{2}$

O questionário, formulado ad hoc para esta pesquisa, permitiu obter indicações sobre os diferentes aspectos da vida e da sensibilidade dos jovens. As respostas foram elaboradas por meio do software Spss 13.0.

Nomeadamente, os itens têm a ver com:

1. A gestão do seu tempo livre, o grau de participação e de pertença no âmbito territorial onde vivem, as sugestões às instituições para a criação de uma cidade mais "à medida de adolescente", a confiança nas próprias instituições, a avaliação dos problemas relativos à zona de residência (Área 1: o contexto urbano: representações, lugares, tempos, necessidades, oportunidades);

2. A relação com os seus pais, as modalidades de expressão e manifestação do seu mal-estar, as prioridades e os valores da vida, a percepção da sua geração (Área 2: Mal-estar, família e pares: representação e relação, expressão emotiva).

\section{Análise dos dados e resultados}

\section{Área 1: contexto urbano: representações, lugares, tempos, necessidades e oportunidades}

Esta primeira área recolhe informações relativas aos hábitos, ao sentido de pertença e inclusão social, às necessidades sociais, em relação a um específico contexto territorial e cultural. ${ }^{3}$ Tentou-se também perceber quais são os valores e as prioridades da vida dos jovens, bem como a percepção que eles têm da sua própria geração.

Nas suas respostas, os adolescentes indicam como lugares habituais de encontro a praça/estrada (57\%), os bares/restaurantes 
fast-food (14,5\%), nenhum lugar específico (17\%), os clubes noturnos e as casas particulares (5\%), os círculos recreativos e cultural $(1,5 \%)$ [Chi-quadro $=19,595 ; \mathrm{df}=5 ; \mathrm{p}=.001]$.

Quadro 1 - Locais habituais de encontro de adolescentes dos municípios do âmbito territorial

Salerno 1

\begin{tabular}{l|l|l|l}
\hline \multirow{2}{*}{$\begin{array}{l}\text { LOCAIS HABITUAIS DE } \\
\text { ENCONTRO }\end{array}$} & SEXO & F & TOTAL \\
\cline { 2 - 4 } Praça/estrada & 51 & 63 & 114 \\
\hline Bares/fast-food & 22 & 7 & 29 \\
\hline Clubes noturnos & 9 & 1 & 10 \\
\hline Círculos recreativos e cultural & 1 & 2 & 3 \\
\hline Casas particulares & 3 & 7 & 10 \\
\hline Nenhum lugar específico & 14 & 20 & 34 \\
\hline TOTAL & 100 & 100 & 200 \\
\hline Fonte: & & & \\
\hline
\end{tabular}

Fonte: elaboração própria.

Os adolescentes desenvolvem as suas atividades de tempo livre, sobretudo, em companhia (93\% rapazes - $81 \%$ meninas) quase sempre na sua própria aldeia (45,5\%) ou sempre $(23,5 \%)$, ou ainda apenas poucos dias por semana (11\%) por algumas horas (4,5\%) [Chi-quadro $=5,444 ; \mathrm{df}=1 ; \mathrm{p}=.020]$.

Quadro 2 - Atividades de tempo livre realizadas por adolescentes dos municípios do Âmbito territorial Salerno 1

\begin{tabular}{l|l|l|l}
\hline \multirow{2}{*}{ ATIVIDADES DE TEMPO LIVRE } & \multicolumn{2}{l}{ SEXO } & TOTAL \\
\cline { 2 - 4 } & M & F & 47 \\
\hline Sempre na sua própria aldeia & 23 & 24 & 91 \\
\hline Quase sempre na sua própria aldeia & 46 & 45 & 22 \\
\hline Apenas poucos dias por semana & 11 & 11 & 9 \\
\hline Por algumas horas & 6 & 3 & 31 \\
\hline Quase nunca, eu passo sempre & 14 & 17 & 200 \\
\hline TOTAL & 100 & 100 & \\
\hline
\end{tabular}

Fonte: elaboração própria.

Eles se reconhecem na pertença territorial à região da Campania e à Itália (13,5\%), ao Município de residência (17\%), à Europa (16,5\%), à Itália do Sul (16\%), ao Agro Nocerino Sarnese (11\%) [Chi-quadro $=16,851 ; \mathrm{df}=7 ; \mathrm{p}=.018]$. 
Quadro 3 - Pertença territorial de adolescentes dos municípios do âmbito territorial Salerno 1

\begin{tabular}{l|l|l|l}
\hline \multirow{2}{*}{ PERTENÇA TERRITORIAL } & SEXO & TOTAL \\
\cline { 2 - 4 } & M & F & 20 \\
\hline Distrito de residência & 9 & 11 & 34 \\
\hline Municipio de residência & 15 & 19 & 22 \\
\hline Agro N-S âmbito S1 & 19 & 3 & 32 \\
\hline Itália do Sul & 13 & 19 & 27 \\
\hline Itália & 12 & 15 & 33 \\
\hline Europa & 14 & 19 & 5 \\
\hline Mundo & 3 & 2 & 27 \\
\hline Campania & 15 & 12 & 200 \\
\hline TOTAL & 100 & 100 &
\end{tabular}

Fonte: elaboração própria.

Sentem-se prevalentemente inseridos na vida da cidade $(76 \%)$ e participantes (72\%), e em medida inferior distantes $(16,5 \%)$ ou excluídos (10\%) [Chi-quadro =4,580; $\mathrm{df}=4 ; \mathrm{p}=.333$ ].

Quadro 4 - Sentimento de inserção na vida da cidade relatado por adolescentes dos municípios do Âmbito territorial Salerno 1

\begin{tabular}{l|l|l|l}
\hline \multirow{2}{*}{ INCLUSÃO/INTEGRAÇÃO } & \multicolumn{2}{|l}{ SEXO } & TOTAL \\
\cline { 2 - 4 } & M & F & 42 \\
\hline Muito & 25 & 17 & 110 \\
\hline Suficiente & 48 & 62 & 35 \\
\hline Pouco & 20 & 15 & 9 \\
\hline De jeito nenhum & 5 & 4 & 4 \\
\hline Eu não sei & 2 & 2 & 200 \\
\hline TOTAL & 100 & 100 & \\
\hline
\end{tabular}

Fonte: elaboração própria.

As expectativas sobre o futuro do seu país aparecem positivas (77\%), sem a previsão de grandes mudanças (10\%), ao passo que uma pequena parte prevê um futuro pior $(0,5 \%)$ [p > .05].

À administração municipal pedem intervenções no âmbito do emprego (37,5,\%); desportivo (36\%) e cultural (8\%), através de trocas e experiências culturais ao estrangeiro (9,5\%). [Chi-quadro $=26,146 ; d f=6 ; p=.000)]$. 
Quadro 5 - Mudanças requisitadas à administração por adolescentes dos municípios do âmbito territorial Salerno 1

\begin{tabular}{|c|c|c|c|}
\hline \multirow{2}{*}{ MUDANÇAS REQUISITADAS } & \multicolumn{3}{|c|}{ SEXO } \\
\hline & M & $\mathbf{F}$ & TOTAL \\
\hline Esporte & 9 & 11 & 20 \\
\hline Cultura e lazer & 15 & 19 & 34 \\
\hline Experiências culturais ao estrangeiro & 19 & 3 & 22 \\
\hline Emprego & 13 & 19 & 32 \\
\hline Habitação (contribuições e benefícios) & 12 & 15 & 27 \\
\hline $\begin{array}{l}\text { Facilitar o acesso aos serviços e } \\
\text { centros culturais, educacionais }\end{array}$ & 14 & 19 & 33 \\
\hline Descontos/convenções & 3 & 2 & 5 \\
\hline TOTAL & 100 & 100 & 200 \\
\hline
\end{tabular}

Fonte: elaboração própria.

A fim de incrementar a relação com os coetâneos, é exigida a criação de estruturas recreativas, culturais e desportivas no território (35\%), centros de agregação juvenil (26,5\%), áreas para concertos e manifestações $(16,5 \%)$, centros comerciais (9\%), parques e zonas verdes públicas $(12,5 \%)$ [Chi-quadro $=9,392 ; d f=4 ; p=.052$ ].

No que diz respeito à confiança nas figuras institucionais, são indicadas as forças da ordem (61,5\%), padres e voluntários (61\%), os quais têm em comum a percepção geral de uma atividade sem fins lucrativos que exige dedicação e generosidade; a seguir encontram-se os professores $(58,5 \%)$ e os órgãos de informação $(50,5 \%)$, juízes (38,5\%), políticos (31,5\%), administradores municipais $(24,5 \%)$ [p $>.05]$.

A própria geração é descrita como sonhadora (71\%), curiosa $(79,5 \%)$, indecisa (70\%), evidenciando a sua dimensão de valores (58\%): concretude (58,5\%), empenho (55\%) solidariedade (54,5\%), e responsabilidade (52\%) [p > .05].

\section{Área 2. mal-estar, família e pares: representação e relação, expressão emotiva}

A segunda área do questionário apresentava uma série de perguntas destinadas a recolher informações sobre as modalidades que os jovens utilizam para viver a sua relação com os pais e as atitudes dos pais em relação a eles. Tentou-se, ainda, perceber 
como estes adolescentes expressam e manifestam o seu mal-estar e a quem, nestes casos, pedem consolo e ajuda.

A partir das respostas percebe-se que os adolescentes podem achar a relação com os seus pais normal (50,2\%), de cumplicidade $(17,5 \%)$, conflitual $(16,5 \%)$ dependente (5\%), de autonomia (7\%) [Chi-quadro 20,561; $d f=5 ; p=.001$ ].

Quadro 6 - Tipos de relações com os pais relatados por adolescentes dos municípios do âmbito territorial Salerno 1

\begin{tabular}{|c|c|c|c|}
\hline \multirow{2}{*}{ PAIS } & \multicolumn{3}{|c|}{ SEXO } \\
\hline & $\mathbf{M}$ & $\mathbf{F}$ & TOTAL \\
\hline Dependente & 3 & 7 & 10 \\
\hline Normal & 47 & 57 & 104 \\
\hline Cumplicidade & 13 & 22 & 35 \\
\hline Autonomia & 13 & 1 & 14 \\
\hline Conflitual & 21 & 12 & 33 \\
\hline Outras & 3 & 1 & 4 \\
\hline TOTAL & 100 & 100 & 200 \\
\hline
\end{tabular}

Fonte: elaboração própria.

As regras em família são estabelecidas por ambos os pais $(41,5 \%)$, por nenhum em particular $(21,5 \%)$, ou por meio de uma discussão conjunta (14\%) [Chi-quadro10,460; $\mathrm{df}=4 ; \mathrm{p}=.033$ ].

Quadro 7 - Formas de estabelecimento de regras em famílias de adolescentes dos municípios do âmbito territorial Salerno 1

\begin{tabular}{l|l|l|l}
\hline \multirow{2}{*}{ REGRAS } & \multicolumn{2}{l}{ SEXO } & TOTAL \\
\cline { 2 - 4 } & M & F & 28 \\
\hline Discussão conjunta & 13 & 15 & 83 \\
\hline Ambos os pais & 39 & 44 & 29 \\
\hline Pai & 19 & 10 & \multicolumn{2}{|l}{27} \\
\hline Mãe & 3 & 14 & 43 \\
\hline Nenhum em particular & 26 & 17 & 200 \\
\hline TOTAL & 100 & 100 & 2 \\
\hline
\end{tabular}

Fonte: elaboração própria.

Os jovens sentem-se bastante controlados pelos pais $(44,5 \%)$, muito $(22,5 \%)$, em medida inferior pouco $(28,5 \%)$ ou nada $(21,5 \%)$. Sentem-se bastante entendidos pelos pais (53\%), muito $(19,5 \%)$, pouco (21\%) ou nada (6,5\%) [Chi-quadro1,037; $d f=3 ; p=.792]$.

Em família gostariam de ter uma maior disponibilidade de dinheiro (51,1 \% M - 35,9\% F) e mais consideração (31,1\% M - 44,9\% 
F), quase ao contrário do que foi afirmado anteriormente (o fato de eles se sentirem entendidos pelos pais) e mais carinho $(5,5 \%$ M - 14,6 F). Muito escasso, no caso das meninas, o pedido de uma maior orientação e firmeza (2,2\%), em confirmação do fato de que elas já se sentem demasiado controladas, em relação aos rapazes $(7,7 \%)$ [Chi-quadro15,885; $\mathrm{df}=4 ; \mathrm{p}=.003]$.

\begin{tabular}{|c|c|c|c|}
\hline \multirow{2}{*}{ DESEJO } & \multicolumn{3}{|c|}{ SEXO } \\
\hline & $\mathbf{M}$ & $\mathbf{F}$ & TOTAL \\
\hline Mais consideração & 27 & 42 & 69 \\
\hline Disponibilidade de dinheiro & 42 & 31 & 73 \\
\hline Maior orientação e firmeza & 7 & 2 & 9 \\
\hline Mais proteção & 3 & 2 & 5 \\
\hline Mais carinho & 1 & 11 & 12 \\
\hline TOTAL & 80 & 88 & 168 \\
\hline
\end{tabular}

Fonte: elaboração própria.

No que diz respeito a percepção da atitude dos adultos em relação ao adolescente, é indicada a confiança nas suas capacidades (82\% M - 87\% F), o respeito da sua maneira de ser jovem (75\% $\mathrm{M}$ e $79 \% \mathrm{~F})$, o fato de o encorajarem nas suas escolhas (72\% $\mathrm{M}$ e $75 \%$ F), embora, manifestando a sua ambivalência, os pais tentem evitar-1he problemas (67\% M e 68\% F), tendo a expectativa de que seja igual a eles (48\% M -31\% F), comparando-o sempre com alguém (44\% M - 35\% F).

Quando vivem um problema ou uma forte dificuldade os jovens voltam-se para os seus coetâneos e a família $(46,1 \%)$ na qual a mãe ocupa o lugar de conselheira (30,5\% F - 15,3\% M) e também os irmãos e as irmãs desempenham um papel importante $(14,2 \%$ M - 15,7\% F) em relação ao pai (6,5\% M - 1,1\% F) e ao parceiro $(4,3 \% \mathrm{M}-3,1 \% \mathrm{~F})$ [Chi-quadro $=67,358 ; \mathrm{df}=12 ; \mathrm{p}=.000]$.

Um outro dado que merece reflexão tem a ver com os professores, que, no caso das meninas (37\%), representam um eventual ponto de referência, mais do que para os rapazes $(6,5 \%)$. As meninas expressam tal mal-estar sobretudo chorando (36\%) ou ainda falando (18\%); enquanto os rapazes teriam dificuldade em o expressar (13\%) e apenas uma pequena percentagem (23\%) fala com alguém ou reage de forma transgressiva (15\%) [Chi-quadro = 35,105; $\mathrm{df}=10 ; \mathrm{p}=.000]$. 
Em conclusão, quase todos os entrevistados consideram importante para a sua vida a família, a amizade e o amor (98\% M - 96\% F); procuram diversão, divertimento, ter uma vida confortável e abastada bem como alcançar a realização pessoal (85\% M - 90\% F), o trabalho (96\% M- 95\% F), a carreira e o sucesso (89\% M - 87\% F). Alguma diferença significativa entre os dois grupos nota-se nas respostas relativas ao estudo (54\% M - $72 \% \mathrm{~F})$ e à cultura $(63 \%$ M - 82\% F), em confirmação do fato que as meninas demonstram mais interesse para o conhecimento, enquanto os rapazes têm a tendência a privilegiar as atividades práticas, como por exemplo, o esporte (90\% M - 63\% F). Nota-se, todavi, o regresso a um equilíbrio de respostas entre os dois grupos em relação à liberdade e democracia (83\% M - 83\% F), justiça, paz, solidariedade (84\% M e $88 \%$ F), igualdade social ( $88 \%$ M - 87\% F), o que demonstra que eles tendem a valorizar o empenho em âmbito social.

\section{Reflexões conclusivas e perspectivas de intervenção}

A partir das representações partilhadas pelos entrevistados (expressas pelas respostas fornecidas aos itens), tentou-se individuar um perfil de adolescente. O resultado foi a sua maneira de entender e viver o contexto territorial, afirmando os seus direitos/deveres de cidadania, também em termos de civicness, ou seja, de orientação para o bem comum caraterizada por atitudes cooperativas e de confiança interpessoal (ALMOND; VERBA, 1980; MAZZOLENI, 2004). Trata-se de adolescentes que não são passivos, mas sim cheios de potencialidades e carga vital. Reconhecem-se e identificam-se no âmbito territorial de proveniência, onde se sentem inseridos e participantes. Colocam na escala dos seus valores de referência a família, a amizade, o amor, a solidariedade, a sinceridade. Acham o lar doméstico um lugar onde se sentem seguros, um ponto de referência onde podem encontrar abrigo em caso de dificuldade, mas movem-se muito no território, da rua à praça, em locais e pontos de encontro. Passam o seu tempo livre prevalentemente em companhia e na sua própria aldeia, vivendo quase sempre em grupo. São altamente sociáveis, flexíveis, capazes de se comunicar e de se adaptar. Pedem às instituições intervenções para melhorar a sua condição social e lugares para aumentar a socialização, as oportunidades culturais, formativas e profissionais, manifestando uma suficiente confiança nas forças da ordem, nos padres, nos vo- 
luntários/operadores sociais e nos professores. Na ambivalência da sua idade, reconhecem-se como sonhadores, curiosos e indecisos, mas ao mesmo tempo como concretos, responsáveis e com valores civis, considerando importantes para a sua vida a liberdade, a democracia, a justiça, a paz, a solidariedade e a igualdade social. A esse respeito, a literatura sobre este assunto confirma as nossas suposições afirmando que a influência protetora da civicness pode ser explicada (também) em consideração de algumas variáveis socioeconômicas que caraterizam o território (GATTI; TREMBLAY; SCHADEE, 2007), constituindo um fator capaz de influenciar positivamente os processos de socialização e a assunção de atitudes pró-sociais (contrários ao desvio comportamental) baseados no empenho e no investimento social (BOBEK et al., 2009).

Estes adolescentes procuram um diálogo com o adulto, utilizam uma linguagem variada, articulada, significante, à procura de uma relação séria e de um reconhecimento sem preconceitos e, na síntese entre influências macroculturais (web, media) e microculturais (familiares e territoriais), redefinem representações e modalidades de ação, demonstrando-se suficientemente orientados para os outros e respeitosos do contexto de proveniência, manifestando uma atitude civil.

No que diz respeito às representações relativas à família e às suas dinâmicas, os adolescentes parecem viver uma relação bastante positiva com os pais, nos quais reconhecem um ponto de referência nos momentos de dificuldade. O que resulta é a imagem de uma geração adulta que demonstra interesse pelos mais jovens e que está disposta a os apoiar, embora com algumas ambivalências implícitas: os adultos envolvem os adolescentes nas decisões, estabelecem com eles as regras a respeitar, favorecem o diálogo e o confronto, encorajam as atitudes autônomas, mas também apresentam pedidos e limites controlando o seu comportamento e esperando uma adaptação às suas expectativas (também em termos de identificação) e tentando evitar os problemas.

No que tange à expressão do mal-estar os entrevistados, nos momentos de dificuldade, falam com os pais, sobretudo com a mãe. Os rapazes têm uma maior tendência para violar normas e regras, bebendo, fumando, andando de moto em alta velocidade, mas em geral afirmam ter dificuldade em expressar "verbalmente" o seu mal-estar, e, portanto muitas vezes fecham-se neles próprios; as meninas, por outro lado, choram, escrevem, falam com os outros: 
duas maneiras diferentes de expressar o seu mal-estar que refletem a educação e a cultura adquirida: a ideia do homem "que não precisa de nada", frio e duro à frente das dificuldades, e a ideia da mulher "fraca" que chora e que desabafa: os típicos aspectos da cultura mediterrânea que ainda estão muito presentes.

Em síntese, achamos que a contribuição dos adolescentes entrevistados pode ser interpretada como um pedido explícito, à sociedade dos adultos, de integração social, de uma maior sensibilização da opinião pública quanto aos temas do mal-estar social (ligado a necessidades insatisfeitas, mas exigíveis) a todos os níveis, evidenciando a necessidade de uma programação social, política e econômica adequada e indispensável para garantir condições de bem-estar social, também aos que devem encarar um transitório sofrimento, ligado às necessidades fisiológicas e às "normais" tarefas de desenvolvimento da sua idade.

A intenção desta pesquisa foi a de iniciar um processo cognitivo destinado não só ao estudo destes assuntos, mas também à regulação de intervenções de sensibilização, informação e prevenção dirigidas à comunidade educativa (contextos formais e informais) a fim de estruturar, da forma mais eficaz, percursos formativos e serviços para adolescentes que sejam mais idôneos às suas verdadeiras exigências e às da comunidade à qual pertencem.

A pergunta é, portanto, se existem intervenções capazes de melhorar os processos de crescimento dos adolescentes a fim de reduzir em primeiro lugar as formas de mal-estar dos adolescentes. A resposta é que as intervenções contra os fatores que, em conjunto, determinam as condições de mal-estar, estão inseridas em dois âmbitos: o primeiro âmbito tem a ver com uma série de ações destinadas a realizar uma diferente organização dos serviços territoriais, com muito mais cuidado para o adolescente e o papel que este desempenha na sociedade, o segundo tem a ver com os percursos educativos/formativos, quer dedicados aos próprios adolescentes, quer às pessoas que têm a responsabilidade de orientá-los e de construir os seus modelos de referência.

O processo de educação/formação não deve ser avaliado apenas em relação à aquisição de conhecimentos e de capacidades por parte dos destinatários: a sua eficácia não deve ser medida nos indivíduos, mas sim como feedback da experiência diária, feedback que modifica as expectativas e os objetivos. As organizações que se ocupam de intervenções sociais (municípios, Planos de zona, 
Terceiro setor etc.) podem ser imaginadas como um organismo no qual todos os operadores (órgãos) interagem, se integram e se influenciam mutuamente, facilitando o processo de desenvolvimento da autonomia dos jovens e de quem mais necessita de apoio.

\title{
Malaise of adolescence: transition and identity contexts of social interaction between culture and education
}

\begin{abstract}
This investigation aims to understand the different social meanings that are created through the systematization of knowledge, within the actual production sites, in social agencies, in real contexts of interaction, whenever individual and regulatory factors, as well as representations and culture (symbolic mediation) become intertwined; it also aims to analyze the descriptive content of representation, the network of social meanings related to interactive and situational contexts of activation and re-production of a belief system. Its goal is to become aware of the perception that teens have of their condition in their own living areas, trying to figure out the elements of discomfort inherent to the process of adapting to change, both from a personal and a contextual point of view. We tried to highlight the social representations that dot the collective imagination of the young and that are affected by their cultural environment, in order to understand how this world is seen, known, perceived, discussed and communicated during the transitional phase of identity in adolescence. To this aim we chose to use a semi-structured questionnaire administered to a sample of 200 teenagers; the questionnaire investigated various areas of life and representations, taking into account the fundamental socio-relational and emotional-affective contextual variables involved in the identity transition. Although cognitive research is not sufficiently exhaustive, as it is limited to the exploration and description of responses, it can be stimulating and useful for practical applications (both in prevention and education); it means to represent a further opportunity, in terms of reflection and encouragement, for those that are in various ways involved in operational and professional contexts, in the joint effort to support adolescents during their delicate, and often difficult, developmental process.
\end{abstract}

Keywords: Adolescence. Malaise. Cultural Contexts. Education.

\section{Referências}

ALMOND, G. A.; VERBA, S. The Civic culture revisited: an analytic study. London: Sage, 1980.

BOBEK, D. et al. Cognitive, emotional and behavioural components of civic action: Towards an integrated measure of civic engagement. Journal of Applied Developmental Psychology, v. 30, n. 5, p. 615-627, 2009.

BRUNER, J. S. In search of mind: essay in autobiography. Michigan: Harper \& Row, 1983.

. Actual minds, possible worlds. Cambridge, MA: Harvard

University Press, 1986. 
1990.

Acts of meaning. Cambridge, MA: Harvard University Press,

A narrative model of self-construction. In: SNODGRASS, J. G.;

Il pensiero, strategie e categorie. Roma: Armando editore. In:

CESARO, M.(2010). Adolescenza e salute mentale. Rappresentazioni del disagio psico-sociale. Milano: Franco Angeli, 2009.

THOMPSON, R. L. (Ed.). Annals of the New York Academy of Sciences, v. 818, 1997.

COLE, M. Cultural Psychology. Cambridge, Mass.: Harvard University Press, 1996.

DE ROSA, A. S. Per un approccio multimetodo allo studio delle rappresentazioni sociali. In: Rassegna di psicologia 3, VII, p. 101-152, 1990.

ERIKSON, E. Gioventù e crisi di identità. Roma: Armando editore, 1974.

FASANO, O. La narrazione autobiografica tra disagio e paure adolescenziali. Roma: Aracne, 2007.

FASANO, O. Rappresentazione sociale e comunicazione del disagio giovanile. Roma: Aracne, 2009.

GATTI, U.; TREMBLAY, R. E.; SCHADEE, H. Civic community and violent behavior in Italy. Aggressive Behavior, v. 33, p. 56-62, 2007.

HAVINGHURST, R. J. Human Development and Education. New York: Longman, 1952.

IANNACCONE, A.; SMORTI, A. Editoriale. Psicologia Culturale, gennaio/ giugno 2009. Roma: Carlo Amore, 2009.

MAZZOLENI, O. Culture politiche e culture civiche a confronto.In: Aspetti Statistici, 16. Ufficio di statistica, Bellinzona, 2004.

MCADAMS, D. P. Personality, modernity, and storied self: a contemporary framework for studying persons. Psychological Inquiry, v. 7, n. 4, p. 295-321, 1996.

MONDA P.; BUONO T. a cura di. La costruzione del benessere nella valle del Sarno, $1^{\circ}$ Rapporto di ricerca e valutazione sulla condizione sociale nell'Ambito territoriale S1 - 2011. Salerno: Piano di Zona S1/ PrintArt, 2011.

MOSCOVICI, S. The phenomenon of social representations. In: FARR, R.M.; MOSCOVICI, S. (Ed.). Social representations. Cambridge:

Cambridge University Press, 1984. (tr. it. 1989, Rappresentazioni sociali,. Bologna: Il Mulino)

SKOWRONSKI, J. J.; WALKER, W. R. How describing autobiographical events can affect autobiographical memories. Social Cognition, v. 22, n. 5, p. 555-590, 2004.

SMORTI, A. Il Sé come testo. Firenze: Giunti, 1997. 
VYGOTSKIJ, L. S. Pensiero e linguaggio. Bari: Laterza, 1934.

VYGOTSKY, L. S. Thought and language. New York: Wiley, 1962.

VYGOTSKIJ, L. S. Storia dello sviluppo delle funzioni psichiche superiori. Firenze: Giunti Barbera, 1981.

Recebido: 25/04/2013 | Aprovado: 22/11/2013 
